\title{
In vitro Multiplication of Banana CV. Rajapuri Bale (Musa spp., AAB Group)
}

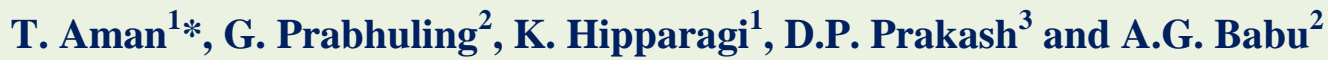 \\ ${ }^{1}$ Department of Fruit Science, College of Horticulture, UHS, Bagalkot, ${ }^{2}$ Directorate of \\ Research, UHS, Bagalkot, India \\ ${ }^{3}$ Department of Fruit Science, College of Horticulture, Munirabad, Koppal dist., India \\ *Corresponding author
}

\section{A B S T R A C T}

\begin{tabular}{|c|c|}
\hline Key & $\begin{array}{l}(\mathrm{AAB}) \text { is a popular cultivar of banana grown in } \\
\text { in Bagalkot, Bijapur and Belgaum. The demand for }\end{array}$ \\
\hline $\begin{array}{l}\text { Rajapuri Bale, } \\
\text { Multiple shoot } \\
\text { clumps, Shoot } \\
\text { proliferation, } \\
\text { Cytokinins }\end{array}$ & $\begin{array}{l}\text { the planting material of this cultivar is high and tissue cultured plantlets is not available } \\
\text { because of the inherent problem in the initial establishment of the culture and } \\
\text { multiplication rate is also low. For facilitating large scale multiplication of this cultivar, the } \\
\text { present investigation was carried to out to optimize shoot proliferation with 6- } \\
\text { Benzylaminopurine (BAP), Thiadiazuron (TDZ) and 6-Benzylaminopurine (BAP) with } \alpha \text { - }\end{array}$ \\
\hline Articl & 11 \\
\hline $\begin{array}{l}\text { Accepte } \\
24 \text { June } \\
\text { Availab } \\
10 \text { July }\end{array}$ & $\begin{array}{l}\text { cytokinins. Among different cytokinins, BAP } 4 \mathrm{mg} / \mathrm{l} \text { was recorded as the best } \\
\text { concentration for shoot growth parameters like per cent regeneration }(100 \%) \text {, days taken } \\
\text { for bud sprouting }(5.41) \text {, number of shoots per explant }(3.13) \text {, number of leaves per shoot } \\
(2.07) \text {, shoot length }(4.72 \mathrm{~cm}) \text {, chlorophyll content and fresh weight of the plantlets } \\
(3374.80 \mathrm{mg} / \text { plantlet) for banana cv. Rajapuri Bale. }\end{array}$ \\
\hline
\end{tabular}

\section{Introduction}

Banana and Plantains (Musa spp.) are some of the earliest crop plants having been domesticated by humans. Bananas are consumed as ripe fruit, whereas plantains, which remain starchy even when fully ripe, need cooking for palatability and consumption. Banana and plantain are among the world's major food crops, and are considered as the poor man's fruit crop in tropical and subtropical countries. They belong to the family Musaceae and section Eumusa with chromosome number 22, 33, 44. The cultivated edible banana is mainly triploid and its basic chromosome number is 11 (Salaria, 2004). It is considered as the symbol of 'prosperity and fertility' owing to its greater socio-economic significance and multifaceted uses and high economic returns it is referred to as "Kalpatharu" (a plant of virtues) and "Kalpavriksh" (Singh, 2009). Rajapuri Bale (AAB) is a popular cultivar of banana grown in Northern parts of Karnataka. It is a dwarf 
variety grows upto 6-8 feet height with a very thick stem and stands up very well to wind. The leaves are wider than those of most bananas growing upto 3 feet wide. The bunches weigh about $10-15 \mathrm{~kg}$ with $8-10$ hands and 90-100 fingers. Fruits are of medium size having attractive yellow colour with thick skin and good blend of sweet and acidity (Rathod, 2013).

Bananas are generally propagated vegetatively through suckers. Unfortunately, the traditional methods limited the expansion of bananas production due to a shortage of healthy plant material availability to farmers. High sterility of most cultivated bananas has historically prevented conventional breeding programs and plant propagation. The major limitation with sucker propagation is the transmission of harmful insects, nematodes and viral diseases to field grown suckers. To overcome these issues and enable rapid multiplication of economically important commercial varieties, in vitro propagation is a preferred alternative method. Shoot tip culturing for bananas, provides second advantages that coincide with the farmers demands including, increased multiplication rate, physiological uniformity and the availability of disease-free materials all year round (Onuoha et al., 2011).

However, due to the presence of the 'B' component in the genotype of Rajapuri Bale (AAB) it adversely affected multiplication especially initially; the more 'B' genomes in the group, the lower the rate of multiplication (Hirimburegama and Gamage, 1997). The rate of multiplication also appears to be related to the degree of browning of the shoot tip tissues, in general, cultivars with 'B' genome showed more tissue browning than those with 'A' genome. The browning effect shown by 'B' genome due to oxidation of phenolic compounds, death of tissues may occur and affect shoot multiplication (Banerjee et al., 1986). Despite having many desirable attributes, this elite native cultivar is not being commercially cultivated mainly due to the inherent problems associated with them such as higher degree of contamination, culture browning, poor multiplication rates, etc., under in vitro conditions. Therefore, present study was undertaken for the standardization of a suitable micropropagation protocol to obtain high rate of multiplication.

\section{Materials and Methods}

\section{Explant preparation}

The explants were collected from healthy and vigorously growing mother plant of the banana cultivar Rajapuri Bale (AAB) grown at mother block, Main Horticulture Research and Extension Centre, University of Horticultural Sciences, Bagalkot, Karnataka. The suckers were washed thoroughly in running tap water to remove the adhering soil particles followed by washing in soap water solution for 30 minutes. Using stainless steel knife, outer leaf sheaths, the leaf bases and rhizome tissues were trimmed away until the length of the shoot was 4-6 cm. The explants were immersed in $1 \%$ Bavistin solution for 30 minutes, again explant were trimmed and immersed in $0.50 \%$ Bavistin $+0.05 \%$ streptocycline solution for 8 hours. Bavistin and K-cycline was discarded and explants were washed thoroughly with distilled water. Plant materials were trimmed again, so that trimmed suckers were of $2-3 \mathrm{~cm}$ in length and $2-2.5 \mathrm{~cm}$ in diameter. Shoot tips were further treated with $0.05 \%$ Citrimide for 30 minutes.

After these treatments, the explants were taken to Laminar Air Flow Chamber (LAF). Again after the removal of one more layer, the explant was treated with $0.10 \%$ mercuric chloride for 10 minutes. Later, they were washed thoroughly for 5-6 times using sterilized double distilled water to remove the traces of mercuric chloride. Later, the shoot 
tips (about $2 \mathrm{~cm}$ ) were excised and inoculated into the culture tubes individually.

\section{Preparation of culture media}

MS (Murashige and Skoog) basal medium, were supplemented with different and various concentrations of plant growth regulators for in vitro multiplication. The, required amounts of growth regulators and or antioxidants were added. The $\mathrm{pH}$ was adjusted to 5.74 by $\mathrm{pH}$ meter using $1 \mathrm{~N} \mathrm{NaOH}$ and $1 \mathrm{~N} \mathrm{HCl}$. After final volume was made up by adding distilled water, the solution was boiled with slow and continuous stirring along with agar at $6.5 \mathrm{~g} / \mathrm{l}$. After the agar was completely dissolved, the media was distributed into Jam bottles of 275 $\mathrm{ml}$ capacity with autoclavable polypropylene caps was used as culture containers for all the experiments, where the media $25 \mathrm{ml} /$ bottle was poured and the bottles with media were sterilized by autoclaving at $121{ }^{0} \mathrm{C}$ temperature and $15 \mathrm{lb}$ pressure for 15 minutes. After sterilization the media was left to cool at room temperature and then kept in inoculation room.

\section{Effect of cytokinin on shoot proliferation}

Multiple shoot clumps were transferred onto multiplication media for shoot multiplication and development. Different concentration of 6-Benzyl aminopurine (BAP), Thidiazuron (TDZ) and Naphthalene acetic acid (NAA) hormones were used for shoot induction and shoot multiplication.

The materials were subcultured twice at a regular interval of four weeks onto same medium to produce multiple shoots. Observations were recorded of percent regeneration, days taken for bud sprouting, number of shoots per explants, length of shoot (cm), number of leaves per shoot, chlorophyll content and fresh weight of plantlets. The regenerated plantlets after developing sufficient root system were carefully removed from the culture vessels. The roots of the plantlets were gently washed under running tap water to remove agar attached to the roots. Immediately after washing they were transferred to protrays containing a sterilized cocopeat and kept under poly tunnel for weeks. Later plantlets transfered to poly bags containing sand, red soil and compost in 1:1:1 ratio $(\mathrm{v} / \mathrm{v})$. They were kept under shade house and sprayed with water regularly to maintain high humidity around the plantlets

\section{Culture conditions}

The cultures were incubated in culture room where a temperature of $25 \pm 2^{0} \mathrm{C}$, relative humidity (RH) of 60 per cent and with photoperiodic cycle of 16 hour light and 8 hour dark conditions (intensity of 2500 lux, fluorescent tubes, Philips, $6500{ }^{\circ} \mathrm{K}, 36$ Watts).

\section{Statistical analysis}

The data were taken at four week intervals after incubation. Data recorded for different parameters were subjected to completely randomized design (CRD). Statistical analysis was done by (ANOVA) using software Wasp developed by ICAR Research Complex, Goa.

\section{Results and Discussion}

\section{Per cent regeneration}

During the first subculture cycle, per cent regeneration of explants was maximum (100 $\%$ ) when multiple shoot bud explants were cultured onto MS media containing BAP 4 $\mathrm{mg} / \mathrm{l}\left(\mathrm{T}_{3}\right)$, BAP $6 \mathrm{mg} / \mathrm{l}+\mathrm{NAA} 0.50 \mathrm{mg} / \mathrm{l}\left(\mathrm{T}_{9}\right)$ and TDZ $0.030 \mathrm{mg} / \mathrm{l}\left(\mathrm{T}_{13}\right)$ followed by BAP 5 $\mathrm{mg} / \mathrm{l}\left(\mathrm{T}_{4}\right)$, BAP $6 \mathrm{mg} / \mathrm{l}\left(\mathrm{T}_{5}\right)$, BAP $3 \mathrm{mg} / \mathrm{l}+$ NAA $0.50 \mathrm{mg} / \mathrm{l}\left(\mathrm{T}_{6}\right)$, TDZ $0.015 \mathrm{mg} / \mathrm{l}\left(\mathrm{T}_{10}\right)$, TDZ $0.020 \mathrm{mg} / \mathrm{l}\left(\mathrm{T}_{11}\right)$ and TDZ $0.035 \mathrm{mg}\left(\mathrm{T}_{14}\right)$ each $93.33 \%$. While, the minimum (66.67) per cent regeneration was observed in control 
$\left(\mathrm{T}_{1}\right)$ followed by BAP $3 \mathrm{mg} / \mathrm{l}(73.33 \%)\left(\mathrm{T}_{2}\right)$. While, in the second subculture cycle significantly maximum (100) per cent regeneration was observed with treatment MS based media supplemented with BAP $4 \mathrm{mg} / \mathrm{l}$ $\left(\mathrm{T}_{3}\right)$ which was followed by BAP $3 \mathrm{mg} / \mathrm{l}\left(\mathrm{T}_{2}\right)$ and TDZ $0.020 \mathrm{mg} / \mathrm{l}\left(\mathrm{T}_{11}\right)$ each $95.83 \%$. Significantly minimum per cent regeneration (66.67) was recorded with MS based media alone $\left(\mathrm{T}_{1}\right)$ which was followed by BAP $5 \mathrm{mg} / \mathrm{l}$ $(82.22 \%)\left(\mathrm{T}_{4}\right)$ and BAP $3 \mathrm{mg} / \mathrm{l}+\mathrm{NAA} 0.50$ $\mathrm{mg} / \mathrm{l}(82.50)\left(\mathrm{T}_{6}\right)$.

\section{Days taken for bud sprouting}

The results of analysed data pertaining to days taken for bud sprouting revealed significant differences for BAP, BAP with NAA and TDZ at first and second subculture cycle. The minimum number of days taken for bud sprouting (5.47) was observed with the treatment MS B + BAP $4 \mathrm{mg} / \mathrm{l}\left(\mathrm{T}_{3}\right)$ which was statistically on par with MS B + BAP $5 \mathrm{mg} / \mathrm{l}$ (5.75 days) $\left(\mathrm{T}_{5}\right)$. Next best treatments were MS B + BAP $6 \mathrm{mg} / \mathrm{l}+\mathrm{NAA} 0.50 \mathrm{mg} / \mathrm{l}(6.20$ days) $\left(\mathrm{T}_{9}\right)$, MS B + BAP $3 \mathrm{mg} / \mathrm{l}$ (6.47 days) $\left(\mathrm{T}_{2}\right)$ and MS B + BAP $6 \mathrm{mg} / \mathrm{l}$ (6.52 days) $\left(\mathrm{T}_{5}\right)$. The maximum days (9.11) for bud sprouting was observed in untreated control $\left(\mathrm{T}_{1}\right)$ followed by MS B + TDZ $0.035 \mathrm{mg} / \mathrm{l}(7.83$ days) $\left(\mathrm{T}_{14}\right), \mathrm{MS} \mathrm{B}+\mathrm{TDZ} 0.020 \mathrm{mg} / \mathrm{l}(7.78$ days) $\left(\mathrm{T}_{11}\right)$ and MS B + TDZ $0.030 \mathrm{mg} / \mathrm{l}(7.67$ days) $\left(T_{13}\right)$. Similarly, during the second subculture cycle significantly minimum number of days taken for bud sprouting (5.35) was recorded in MS B + BAP $4 \mathrm{mg} / \mathrm{l}\left(\mathrm{T}_{3}\right)$ which was on par with MS B + BAP $6 \mathrm{mg} / \mathrm{l}$ (5.49 days) $\left(\mathrm{T}_{5}\right)$ and MS B + BAP $5 \mathrm{mg} / \mathrm{l}(5.51$ days) $\left(\mathrm{T}_{4}\right)$. The maximum days $(8.75)$ for bud sprouting was observed in untreated control $\left(\mathrm{T}_{1}\right)$ followed by MS B + TDZ $0.035 \mathrm{mg} / \mathrm{l}$ (7.13 days) $\left(\mathrm{T}_{14}\right)$ and on par with MS $\mathrm{B}+$ TDZ $0.030 \mathrm{mg} / \mathrm{l}$ (7.07 days) $\left(\mathrm{T}_{13}\right)$ and MS B + BAP $4 \mathrm{mg} / \mathrm{l}+\mathrm{NAA} 0.50 \mathrm{mg} / \mathrm{l}$ (7.01 days) $\left(\mathrm{T}_{7}\right)$.

\section{Number of shoots per explants}

The percural of data pertaining to number of shoots per explant revealed significant differences for different treatments at first and second subculture cycle. Significantly maximum number of shoots per explant (3.20) was observed with the MS B + BAP $4 \mathrm{mg} / \mathrm{l}$ $\left(\mathrm{T}_{3}\right)$ which was statistically on par with MS B + BAP $4 \mathrm{mg} / \mathrm{l}$ + NAA $0.50 \mathrm{mg} / \mathrm{l}(3.07)\left(\mathrm{T}_{7}\right)$. Next best treatments were MS B + BAP 5 $\mathrm{mg} / \mathrm{l}(3.00)\left(\mathrm{T}_{4}\right)$ and $\mathrm{MS} \mathrm{B}+\mathrm{BAP} 6 \mathrm{mg} / \mathrm{l}$ (2.47) $\left(\mathrm{T}_{5}\right)$. While significantly minimum (1.30) number of shoots per explant was observed with control treatment $\left(\mathrm{T}_{1}\right)$ which was on par with MS B + TDZ $0.035 \mathrm{mg} / \mathrm{l}$ (1.32) $\left(\mathrm{T}_{14}\right)$. During the second subculture cycle, the maximum number of shoots per explant (3.06) was observed with MS B + BAP $4 \mathrm{mg} / \mathrm{l}\left(\mathrm{T}_{3}\right)$ which was followed by MS $\mathrm{B}+\mathrm{BAP} 5 \mathrm{mg} / \mathrm{l}(2.81)\left(\mathrm{T}_{4}\right)$ and $\mathrm{MS} \mathrm{B}+\mathrm{BAP}$ $4 \mathrm{mg} / \mathrm{l}+\mathrm{NAA} 0.50 \mathrm{mg} / \mathrm{l}(2.59)\left(\mathrm{T}_{7}\right)$. While, the minimum (1.28) number of shoots per explant was observed with untreated control $\left(\mathrm{T}_{1}\right)$ which was followed by MS B + TDZ $0.035 \mathrm{mg} / \mathrm{l}(2.17)\left(\mathrm{T}_{14}\right)$

\section{Number of leaves per shoot}

During the first subculture cycle, significantly maximum number of number of leaves per shoot (2.00) was observed in BAP $4 \mathrm{mg} / \mathrm{l}\left(\mathrm{T}_{3}\right)$ which was on par with BAP $5 \mathrm{mg} / \mathrm{l}(1.83)\left(\mathrm{T}_{4}\right)$ followed by BAP $4 \mathrm{mg} / \mathrm{l}+\mathrm{NAA} 0.50 \mathrm{mg} / \mathrm{l}$ (1.75) $\left(\mathrm{T}_{7}\right)$. Whereas, minimum number of leaves per shoot (0.88) was observed with MS based medium alone $\left(\mathrm{T}_{1}\right)$ which was followed by TDZ $0.035 \mathrm{mg} / \mathrm{l}(1.23)\left(\mathrm{T}_{14}\right)$ and TDZ $0.025 \mathrm{mg} / \mathrm{l}(1.30)\left(\mathrm{T}_{12}\right)$. While, in the second subculture cycle, significantly maximum number of number of leaves per shoot (2.13) was recorded in treatment BAP $4 \mathrm{mg} / \mathrm{l}\left(\mathrm{T}_{3}\right)$ which was followed by BAP $5 \mathrm{mg} / \mathrm{l}(2.07)\left(\mathrm{T}_{4}\right)$ and BAP $4 \mathrm{mg} / \mathrm{l}+\mathrm{NAA} 0.50 \mathrm{mg} / \mathrm{l}(1.87)\left(\mathrm{T}_{7}\right)$ this was found to be statistically on par with BAP $3 \mathrm{mg} / \mathrm{l}+\mathrm{NAA} 0.50 \mathrm{mg} / \mathrm{l}(1.86)\left(\mathrm{T}_{6}\right)$. The minimum number of leaves per shoot $(0.73)$ 
was observed in untreated control $\left(\mathrm{T}_{1}\right)$ which was followed by TDZ $0.035 \mathrm{mg} / \mathrm{l}(1.20)\left(\mathrm{T}_{14}\right)$ and TDZ $0.030 \mathrm{mg} / \mathrm{l}(1.41)\left(\mathrm{T}_{13}\right)$.

\section{Length of shoot}

In the first subculture cycle, significantly maximum shoot length $(4.02 \mathrm{~cm})$ was recorded in BAP $4 \mathrm{mg} / \mathrm{l}\left(\mathrm{T}_{3}\right)$ followed by BAP $3 \mathrm{mg} / \mathrm{l}(3.68 \mathrm{~cm})\left(\mathrm{T}_{2}\right)$ and BAP $6 \mathrm{mg} / \mathrm{l}+\mathrm{NAA}$ $0.50 \mathrm{mg} / \mathrm{l}(3.60 \mathrm{~cm})\left(\mathrm{T}_{9}\right)$. While, significantly minimum length of shoot $(2.48 \mathrm{~cm})$ was recorded with TDZ $0.035 \mathrm{mg} / \mathrm{l}\left(\mathrm{T}_{14}\right)$ followed by TDZ $0.030 \mathrm{mg} / \mathrm{l}(2.98 \mathrm{~cm})\left(\mathrm{T}_{13}\right)$ and TDZ $0.025 \mathrm{mg} / \mathrm{l}(3.09 \mathrm{~cm})\left(\mathrm{T}_{12}\right)$. During the second subculture cycle, significantly maximum shoot length $(5.33 \mathrm{~cm})$ was recorded with BAP 4 $\mathrm{mg} / \mathrm{l}\left(\mathrm{T}_{3}\right)$ which was followed by BAP $6 \mathrm{mg} / \mathrm{l}$ + NAA $0.50 \mathrm{mg} / \mathrm{l}(4.47 \mathrm{~cm})\left(\mathrm{T}_{9}\right)$ and BAP 4 $\mathrm{mg} / \mathrm{l}+\mathrm{NAA} 0.50 \mathrm{mg} / \mathrm{l}(4.40 \mathrm{~cm})\left(\mathrm{T}_{7}\right)$.
Whereas, the minimum length of shoot (3.23 $\mathrm{cm})$ was recorded with TDZ $0.035 \mathrm{mg} / \mathrm{l}\left(\mathrm{T}_{14}\right)$ which was followed by TDZ $0.025 \mathrm{mg} / \mathrm{l}$ (3.33 $\mathrm{cm})\left(\mathrm{T}_{12}\right)$.

\section{Chlorophyll content}

Significantly maximum content of chlorophyll a $(0.70 \mathrm{mg} / \mathrm{g}$ fresh weight), chlorophyll b $(0.25 \mathrm{mg} / \mathrm{g}$ fresh weight) and total chlorophyll (0.82 $\mathrm{mg} / \mathrm{g}$ fresh weight) was recorded with BAP $4 \mathrm{mg} / \mathrm{l}\left(\mathrm{T}_{3}\right)$ which was followed by BAP $5 \mathrm{mg} / \mathrm{l}\left(\mathrm{T}_{4}\right)$ with chlorophyll a $(0.63 \mathrm{mg} / \mathrm{g}$ fresh weight $)$, chlorophyll $b(0.20 \mathrm{mg} / \mathrm{g}$ fresh weight $)$ and total chlorophyll $(0.77 \mathrm{mg} / \mathrm{g}$ fresh weight). Whereas, minimum content of chlorophyll a $(0.37 \mathrm{mg} / \mathrm{g}$ fresh weight), chlorophyll b $(0.10 \mathrm{mg} / \mathrm{g}$ fresh weight $)$ and total chlorophyll $(0.45 \mathrm{mg} / \mathrm{g}$ fresh weight $)$ was recorded with untreated control $\left(\mathrm{T}_{1}\right)$.

Table.1 Effect of cytokinins on per cent regeneration of banana cv. Rajapuri Bale (AAB)

\begin{tabular}{|c|c|c|c|}
\hline \multirow[b]{2}{*}{ Treatments } & \multicolumn{2}{|c|}{ Per cent Regeneration } & \multirow[b]{2}{*}{ Mean } \\
\hline & $\begin{array}{l}\text { First subculture } \\
\text { cycle }\end{array}$ & $\begin{array}{l}\text { Second subculture } \\
\text { cycle }\end{array}$ & \\
\hline $\mathrm{T}_{1}$ - Full strength MS B* without hormone (Control) & $66.67(54.74) *$ & $66.67(54.74)$ & $66.67(54.74)$ \\
\hline $\mathrm{T}_{2}$ - Full strength MS B + BAP $3 \mathrm{mg} / \mathrm{l}$ & $73.33(59.00)$ & $95.83(78.23)$ & $84.58(66.93)$ \\
\hline $\mathrm{T}_{3}$ - Full strength MS B + BAP $4 \mathrm{mg} / \mathrm{l}$ & $100.00(89.56)$ & $100.00(89.56)$ & $100.00(89.55)$ \\
\hline $\mathrm{T}_{4}$ - Full strength MS B + BAP $5 \mathrm{mg} / \mathrm{l}$ & $93.33(75.24)$ & $82.22(65.05)$ & $87.78(69.55)$ \\
\hline $\mathrm{T}_{5}$ - Full strength MS B + BAP 6 mg/l & $93.33(75.24)$ & $91.11(72.64)$ & $92.22(73.84)$ \\
\hline $\mathrm{T}_{6}$ - Full strength MS B + BAP $3 \mathrm{mg} / \mathrm{l}+\mathrm{NAA} 0.50 \mathrm{mg} / \mathrm{l}$ & $93.33(75.24)$ & $82.50(65.27)$ & $87.92(69.68)$ \\
\hline $\mathrm{T}_{7}$ - Full strength MS B + BAP $4 \mathrm{mg} / \mathrm{l}+\mathrm{NAA} 0.50 \mathrm{mg} / \mathrm{l}$ & $80.00(63.44)$ & $91.67(73.23)$ & $85.83(67.89)$ \\
\hline $\mathrm{T}_{8^{-}}$Full strength MS B + BAP $5 \mathrm{mg} / \mathrm{l}+\mathrm{NAA} 0.50 \mathrm{mg} / \mathrm{l}$ & $86.67(68.67)$ & $90.00(74.85)$ & $88.33(70.54)$ \\
\hline $\mathrm{T}_{9^{-}}$Full strength MS B + BAP $6 \mathrm{mg} / \mathrm{l}+\mathrm{NAA} 0.50 \mathrm{mg} / \mathrm{l}$ & $100.00(89.56)$ & $86.11(68.11)$ & $93.05(74.71)$ \\
\hline $\mathrm{T}_{10^{-}}$Full strength MS B + TDZ $0.015 \mathrm{mg} / \mathrm{l}$ & $93.33(75.24)$ & $93.64(75.38)$ & $93.49(75.49)$ \\
\hline $\mathrm{T}_{11}$ - Full strength MS B + TDZ $0.020 \mathrm{mg} / \mathrm{l}$ & $93.33(75.24)$ & $95.83(78.23)$ & $94.58(76.93)$ \\
\hline $\mathrm{T}_{12}$ - Full strength MS B + TDZ $0.025 \mathrm{mg} / \mathrm{l}$ & $80.00(63.44)$ & $89.68(71.27)$ & $84.84(67.08)$ \\
\hline $\mathrm{T}_{13}$ - Full strength MS B + TDZ $0.030 \mathrm{mg} / \mathrm{l}$ & $100.00(89.56)$ & $92.50(74.10)$ & $96.25(78.83)$ \\
\hline $\mathrm{T}_{14}$ - Full strength MS B + TDZ $0.035 \mathrm{mg} / \mathrm{l}$ & $93.33(75.24)$ & $84.11(67.23)$ & $88.72(71.00)$ \\
\hline S. Em \pm & 2.18 & 2.29 & - \\
\hline $\mathrm{CD}$ at $1 \%$ & 6.35 & 6.68 & - \\
\hline
\end{tabular}

Note - * Figures in parenthesis indicates arc sin transformed values.

MS B-Murashiage and Skoog basal medium 
Table.2 Effect of cytokinins on days taken for bud sprouting in banana cv. Rajapuri Bale (AAB).

\begin{tabular}{|c|c|c|c|}
\hline \multirow[b]{2}{*}{ Treatments } & \multicolumn{2}{|c|}{ Days taken for bud sprouting } & \multirow[b]{2}{*}{ Mean } \\
\hline & $\begin{array}{l}\text { First subculture } \\
\text { cycle }\end{array}$ & $\begin{array}{l}\text { Second subculture } \\
\text { cycle }\end{array}$ & \\
\hline $\mathrm{T}_{1}$ - Full strength MS B* without hormone (Control) & 9.11 & 8.75 & 8.93 \\
\hline $\mathrm{T}_{2}$ - Full strength MS B + BAP $3 \mathrm{mg} / \mathrm{l}$ & 6.47 & 6.13 & 6.30 \\
\hline $\mathrm{T}_{3}$ - Full strength MS B + BAP 4 mg/l & 5.47 & 5.35 & 5.41 \\
\hline $\mathrm{T}_{4^{-}}$Full strength MS B + BAP $5 \mathrm{mg} / \mathrm{l}$ & 5.75 & 5.51 & 5.63 \\
\hline $\mathrm{T}_{5^{-}}$Full strength MS B + BAP $6 \mathrm{mg} / \mathrm{l}$ & 6.52 & 5.49 & 6.00 \\
\hline $\mathrm{T}_{6}$ - Full strength MS B + BAP $3 \mathrm{mg} / \mathrm{l}+\mathrm{NAA} 0.50 \mathrm{mg} / \mathrm{l}$ & 6.97 & 6.70 & 6.83 \\
\hline $\mathrm{T}_{7^{-}}$Full strength MS B + BAP $4 \mathrm{mg} / \mathrm{l}+\mathrm{NAA} 0.50 \mathrm{mg} / \mathrm{l}$ & 7.33 & 7.01 & 7.17 \\
\hline $\mathrm{T}_{8^{-}}$Full strength MS B + BAP $5 \mathrm{mg} / \mathrm{l}+\mathrm{NAA} 0.50 \mathrm{mg} / \mathrm{l}$ & 6.67 & 6.04 & 6.35 \\
\hline $\mathrm{T}_{9}$ - Full strength MS B + BAP 6 mg/l + NAA $0.50 \mathrm{mg} / \mathrm{l}$ & 6.20 & 5.97 & 6.09 \\
\hline $\mathrm{T}_{10^{-}}$Full strength MS B + TDZ $0.015 \mathrm{mg} / \mathrm{l}$ & 6.97 & 6.37 & 6.67 \\
\hline $\mathrm{T}_{11}$ - Full strength MS B + TDZ $0.020 \mathrm{mg} / \mathrm{l}$ & 7.78 & 6.78 & 7.28 \\
\hline $\mathrm{T}_{12}$ - Full strength MS B + TDZ $0.025 \mathrm{mg} / \mathrm{l}$ & 7.39 & 6.80 & 7.09 \\
\hline $\mathrm{T}_{13}$ - Full strength MS B + TDZ $0.030 \mathrm{mg} / \mathrm{l}$ & 7.67 & 7.07 & 7.37 \\
\hline $\mathrm{T}_{14}$ - Full strength MS B + TDZ $0.035 \mathrm{mg} / \mathrm{l}$ & 7.83 & 7.13 & 7.48 \\
\hline S. Em \pm & 0.20 & 0.16 & - \\
\hline $\mathrm{CD}$ at $1 \%$ & 0.60 & 0.47 & - \\
\hline
\end{tabular}

Table.3 Effect of cytokinins on number of shoots per explant in banana cv. Rajapuri Bale (AAB)

\begin{tabular}{|c|c|c|c|}
\hline \multirow[b]{2}{*}{ Treatments } & \multicolumn{2}{|c|}{ Number of shoots per explant } & \multirow[b]{2}{*}{ Mean } \\
\hline & $\begin{array}{l}\text { First subculture } \\
\text { cycle }\end{array}$ & $\begin{array}{l}\text { Second subculture } \\
\text { cycle }\end{array}$ & \\
\hline $\mathrm{T}_{1}$ - Full strength MS $\mathrm{B} *$ without hormone (Control) & 1.30 & 1.28 & 1.29 \\
\hline $\mathrm{T}_{2}$ - Full strength MS B + BAP $3 \mathrm{mg} / \mathrm{l}$ & 2.07 & 2.36 & 2.21 \\
\hline $\mathrm{T}_{3}$ - Full strength MS B + BAP $4 \mathrm{mg} / \mathrm{l}$ & 3.20 & 3.06 & 3.13 \\
\hline $\mathrm{T}_{4}$ - Full strength MS B + BAP $5 \mathrm{mg} / \mathrm{l}$ & 3.00 & 2.81 & 2.91 \\
\hline $\mathrm{T}_{5^{-}}$Full strength MS B + BAP $6 \mathrm{mg} / \mathrm{l}$ & 2.47 & 2.32 & 2.39 \\
\hline $\mathrm{T}_{6}-$ Full strength MS B + BAP $3 \mathrm{mg} / \mathrm{l}+\mathrm{NAA} 0.50 \mathrm{mg} / \mathrm{l}$ & 2.20 & 2.50 & 2.35 \\
\hline $\mathrm{T}_{7}$ - Full strength MS B + BAP $4 \mathrm{mg} / \mathrm{l}+\mathrm{NAA} 0.50 \mathrm{mg} / \mathrm{l}$ & 3.07 & 2.59 & 2.83 \\
\hline $\mathrm{T}_{8^{-}}$Full strength MS B + BAP $5 \mathrm{mg} / \mathrm{l}+\mathrm{NAA} 0.50 \mathrm{mg} / \mathrm{l}$ & 2.00 & 2.21 & 2.10 \\
\hline$T_{9}$ - Full strength MS B + BAP $6 \mathrm{mg} / \mathrm{l}+\mathrm{NAA} 0.50 \mathrm{mg} / \mathrm{l}$ & 1.67 & 2.39 & 2.03 \\
\hline $\mathrm{T}_{10}$ - Full strength MS B + TDZ $0.015 \mathrm{mg} / \mathrm{l}$ & 2.33 & 2.45 & 2.39 \\
\hline $\mathrm{T}_{11}$ - Full strength MS B + TDZ $0.020 \mathrm{mg} / \mathrm{l}$ & 2.00 & 2.29 & 2.14 \\
\hline $\mathrm{T}_{12}$ - Full strength MS B + TDZ $0.025 \mathrm{mg} / \mathrm{l}$ & 1.93 & 2.24 & 2.08 \\
\hline $\mathrm{T}_{13}$ - Full strength MS B + TDZ $0.030 \mathrm{mg} / \mathrm{l}$ & 1.60 & 2.20 & 1.90 \\
\hline $\mathrm{T}_{14}$ - Full strength MS B + TDZ $0.035 \mathrm{mg} / \mathrm{l}$ & 1.32 & 2.17 & 1.74 \\
\hline S. Em \pm & 0.04 & 0.05 & - \\
\hline $\mathrm{CD}$ at $1 \%$ & 0.14 & 0.16 & - \\
\hline
\end{tabular}


Table.4 Effect of cytokinins on number of leaves per shoot in banana cv. Rajapuri Bale (AAB).

\begin{tabular}{|c|c|c|c|}
\hline \multirow[b]{2}{*}{ Treatments } & \multicolumn{2}{|c|}{ Number of leaves per shoot } & \multirow[b]{2}{*}{ Mean } \\
\hline & $\begin{array}{l}\text { First subculture } \\
\text { cycle }\end{array}$ & $\begin{array}{l}\text { Second subculture } \\
\text { cycle }\end{array}$ & \\
\hline $\mathrm{T}_{1}$ - Full strength MS B* without hormone (Control) & 0.88 & 0.73 & 0.81 \\
\hline $\mathrm{T}_{2}$ - Full strength MS B + BAP $3 \mathrm{mg} / \mathrm{l}$ & 1.60 & 1.67 & 1.64 \\
\hline $\mathrm{T}_{3}$ - Full strength MS B + BAP 4 mg/l & 2.00 & 2.13 & 2.07 \\
\hline $\mathrm{T}_{4^{-}}$Full strength MS B + BAP $5 \mathrm{mg} / \mathrm{l}$ & 1.83 & 2.07 & 1.95 \\
\hline $\mathrm{T}_{5}$ - Full strength MS B + BAP 6 mg/l & 1.42 & 1.50 & 1.46 \\
\hline $\mathrm{T}_{6}$ - Full strength MS B + BAP $3 \mathrm{mg} / \mathrm{l}+\mathrm{NAA} 0.50 \mathrm{mg} / \mathrm{l}$ & 1.70 & 1.86 & 1.78 \\
\hline $\mathrm{T}_{7^{-}}$Full strength MS B + BAP $4 \mathrm{mg} / \mathrm{l}+\mathrm{NAA} 0.50 \mathrm{mg} / \mathrm{l}$ & 1.75 & 1.87 & 1.81 \\
\hline $\mathrm{T}_{8^{-}}$Full strength MS B + BAP $5 \mathrm{mg} / \mathrm{l}+\mathrm{NAA} 0.50 \mathrm{mg} / \mathrm{l}$ & 1.53 & 1.57 & 1.55 \\
\hline $\mathrm{T}_{9}$ - Full strength MS B + BAP $6 \mathrm{mg} / \mathrm{l}+\mathrm{NAA} 0.50 \mathrm{mg} / \mathrm{l}$ & 1.43 & 1.62 & 1.53 \\
\hline $\mathrm{T}_{10^{-}}$Full strength MS B + TDZ $0.015 \mathrm{mg} / \mathrm{l}$ & 1.50 & 1.68 & 1.59 \\
\hline $\mathrm{T}_{11}$ - Full strength MS B + TDZ $0.020 \mathrm{mg} / \mathrm{l}$ & 1.47 & 1.50 & 1.48 \\
\hline $\mathrm{T}_{12}$ - Full strength MS B + TDZ $0.025 \mathrm{mg} / \mathrm{l}$ & 1.30 & 1.52 & 1.41 \\
\hline $\mathrm{T}_{13}$ - Full strength MS B + TDZ $0.030 \mathrm{mg} / \mathrm{l}$ & 1.34 & 1.41 & 1.37 \\
\hline $\mathrm{T}_{14}$ - Full strength MS B + TDZ $0.035 \mathrm{mg} / \mathrm{l}$ & 1.23 & 1.20 & 1.22 \\
\hline S. Em \pm & 0.08 & 0.03 & - \\
\hline $\mathrm{CD}$ at $1 \%$ & 0.24 & 0.10 & - \\
\hline
\end{tabular}

Table.5 Effect of cytokinins on length of shoot in banana cv. Rajapuri Bale (AAB)

\begin{tabular}{|l|c|c|c|}
\hline \multirow{2}{*}{ Treatments } & \multicolumn{2}{|c|}{ Length of shoot $(\mathrm{cm})$} & \\
\cline { 2 - 3 } & $\begin{array}{c}\text { First subculture } \\
\text { cycle }\end{array}$ & $\begin{array}{c}\text { Second subculture } \\
\text { cycle }\end{array}$ & Mean \\
\hline $\mathrm{T}_{1}$ - Full strength MS B* without hormone (Control) & 3.15 & 3.62 & 3.38 \\
\hline $\mathrm{T}_{2}$ - Full strength MS B + BAP 3 mg/l & 3.68 & 4.18 & 3.93 \\
\hline $\mathrm{T}_{3}$ - Full strength MS B + BAP 4 mg/l & 4.02 & 5.33 & 4.72 \\
\hline $\mathrm{T}_{4}$ - Full strength MS B + BAP 5 mg/l & 3.29 & 4.27 & 3.78 \\
\hline $\mathrm{T}_{5}$ - Full strength MS B + BAP 6 mg/l & 3.53 & 4.17 & 3.85 \\
\hline $\mathrm{T}_{6}$ - Full strength MS B + BAP 3 mg/l + NAA 0.50 mg/l & 3.51 & 4.00 & 3.75 \\
\hline $\mathrm{T}_{7}$ - Full strength MS B + BAP 4 mg/l + NAA 0.50 mg/l & 3.49 & 4.40 & 3.94 \\
\hline $\mathrm{T}_{8}$ - Full strength MS B + BAP 5 mg/l + NAA 0.50 mg/l & 3.36 & 4.13 & 3.75 \\
\hline $\mathrm{T}_{9}$ - Full strength MS B + BAP 6 mg/l + NAA 0.50 mg/l & 3.60 & 4.47 & 4.03 \\
\hline $\mathrm{T}_{10}$ - Full strength MS B + TDZ 0.015 mg/l & 3.46 & 3.87 & 3.66 \\
\hline $\mathrm{T}_{11}$ - Full strength MS B + TDZ 0.020 mg/l & 3.27 & 3.67 & 3.47 \\
\hline $\mathrm{T}_{12}$ - Full strength MS B + TDZ 0.025 mg/l & 3.09 & 3.33 & 3.21 \\
\hline $\mathrm{T}_{13}$ - Full strength MS B + TDZ 0.030 mg/l & 2.98 & 3.47 & 3.22 \\
\hline $\mathrm{T}_{14}$ - Full strength MS B + TDZ 0.035 mg/l & 2.48 & 3.23 & 2.86 \\
\hline $\mathrm{S}$. Em \pm & 0.06 & 0.08 & - \\
\hline CD at $1 \%$ & 0.19 & 0.23 & - \\
\hline
\end{tabular}


Table.6 Effect of cytokinins on chlorophyll content of the in vitro grown plantlets of banana cv. Rajapuri Bale (AAB)

\begin{tabular}{|c|c|c|c|}
\hline \multirow[b]{2}{*}{ Treatments } & \multicolumn{3}{|c|}{ Chlorophyll Content (mg/g fresh weight) } \\
\hline & Chlorophyll a & Chlorophyll b & Total chlorophyll \\
\hline $\mathrm{T}_{1}$ - Full strength MS B* without hormone (Control) & 0.37 & 0.10 & 0.45 \\
\hline $\mathrm{T}_{2}$ - Full strength MS B + BAP $3 \mathrm{mg} / \mathrm{l}$ & 0.49 & 0.16 & 0.69 \\
\hline $\mathrm{T}_{3}$ - Full strength MS B + BAP $4 \mathrm{mg} / \mathrm{l}$ & 0.70 & 0.25 & 0.82 \\
\hline $\mathrm{T}_{4}$ - Full strength MS B + BAP $5 \mathrm{mg} / \mathrm{l}$ & 0.63 & 0.20 & 0.77 \\
\hline $\mathrm{T}_{5}$ - Full strength MS B + BAP $6 \mathrm{mg} / \mathrm{l}$ & 0.47 & 0.18 & 0.72 \\
\hline $\mathrm{T}_{6}-$ Full strength MS B + BAP $3 \mathrm{mg} / \mathrm{l}+\mathrm{NAA} 0.50 \mathrm{mg} / \mathrm{l}$ & 0.41 & 0.11 & 0.50 \\
\hline $\mathrm{T}_{7}$ - Full strength MS B + BAP $4 \mathrm{mg} / \mathrm{l}+\mathrm{NAA} 0.50 \mathrm{mg} / \mathrm{l}$ & 0.50 & 0.12 & 0.61 \\
\hline $\mathrm{T}_{8^{-}}$Full strength MS B + BAP $5 \mathrm{mg} / \mathrm{l}+\mathrm{NAA} 0.50 \mathrm{mg} / \mathrm{l}$ & 0.52 & 0.13 & 0.63 \\
\hline $\mathrm{T}_{9}$ - Full strength MS B + BAP $6 \mathrm{mg} / \mathrm{l}+\mathrm{NAA} 0.50 \mathrm{mg} / \mathrm{l}$ & 0.52 & 0.14 & 0.66 \\
\hline $\mathrm{T}_{10^{-}}$Full strength MS B + TDZ $0.015 \mathrm{mg} / \mathrm{l}$ & 0.54 & 0.19 & 0.72 \\
\hline $\mathrm{T}_{11}$ - Full strength MS B + TDZ $0.020 \mathrm{mg} / \mathrm{l}$ & 0.46 & 0.15 & 0.60 \\
\hline $\mathrm{T}_{12}$ - Full strength MS B + TDZ $0.025 \mathrm{mg} / \mathrm{l}$ & 0.55 & 0.17 & 0.69 \\
\hline $\mathrm{T}_{13}$ - Full strength MS B + TDZ $0.030 \mathrm{mg} / \mathrm{l}$ & 0.46 & 0.15 & 0.59 \\
\hline $\mathrm{T}_{14}$ - Full strength MS B + TDZ $0.035 \mathrm{mg} / \mathrm{l}$ & 0.38 & 0.14 & 0.48 \\
\hline S. Em \pm & 0.006 & 0.004 & 0.006 \\
\hline $\mathrm{CD}$ at $1 \%$ & 0.016 & 0.012 & 0.016 \\
\hline
\end{tabular}

Table.7 Effect of cytokinins on the fresh weight of plantlets of banana cv. Rajapuri Bale (AAB)

\begin{tabular}{|c|c|}
\hline Treatments & $\begin{array}{c}\text { Fresh weight/ plantlet } \\
\text { (mg/plantlet) }\end{array}$ \\
\hline $\mathrm{T}_{1}$ - Full strength MS $\mathrm{B}^{*}$ without hormone (Control) & 1857.53 \\
\hline $\mathrm{T}_{2}$ - Full strength MS B + BAP $3 \mathrm{mg} / \mathrm{l}$ & 3080.73 \\
\hline $\mathrm{T}_{3}$ - Full strength MS B + BAP $4 \mathrm{mg} / \mathrm{l}$ & 3374.80 \\
\hline $\mathrm{T}_{4}$ - Full strength MS B + BAP $5 \mathrm{mg} / \mathrm{l}$ & 3188.33 \\
\hline $\mathrm{T}_{5}$ - Full strength MS B + BAP 6 mg/l & 3339.00 \\
\hline $\mathrm{T}_{6^{-}}$Full strength MS B + BAP $3 \mathrm{mg} / \mathrm{l}+$ NAA $0.50 \mathrm{mg} / \mathrm{l}$ & 2810.60 \\
\hline $\mathrm{T}_{7}$ - Full strength MS B + BAP $4 \mathrm{mg} / \mathrm{l}+\mathrm{NAA} 0.50 \mathrm{mg} / \mathrm{l}$ & 2936.80 \\
\hline $\mathrm{T}_{8-}$ - Full strength MS B + BAP $5 \mathrm{mg} / \mathrm{l}+$ NAA $0.50 \mathrm{mg} / \mathrm{l}$ & 3009.06 \\
\hline $\mathrm{T}_{9}-$ Full strength MS B + BAP $6 \mathrm{mg} / \mathrm{l}+\mathrm{NAA} 0.50 \mathrm{mg} / \mathrm{l}$ & 2785.60 \\
\hline $\mathrm{T}_{10^{-}}$Full strength MS B + TDZ $0.015 \mathrm{mg} / \mathrm{l}$ & 2824.66 \\
\hline $\mathrm{T}_{11}-$ Full strength MS B + TDZ $0.020 \mathrm{mg} / \mathrm{l}$ & 2562.46 \\
\hline $\mathrm{T}_{12}$ - Full strength MS B + TDZ $0.025 \mathrm{mg} / \mathrm{l}$ & 2707.66 \\
\hline $\mathrm{T}_{13}-$ Full strength MS B + TDZ $0.030 \mathrm{mg} / \mathrm{l}$ & 2496.93 \\
\hline $\mathrm{T}_{14}-$ Full strength MS B + TDZ $0.035 \mathrm{mg} / \mathrm{l}$ & 2433.93 \\
\hline S. Em \pm & 75.28 \\
\hline $\mathrm{CD}$ at $1 \%$ & 219.22 \\
\hline
\end{tabular}




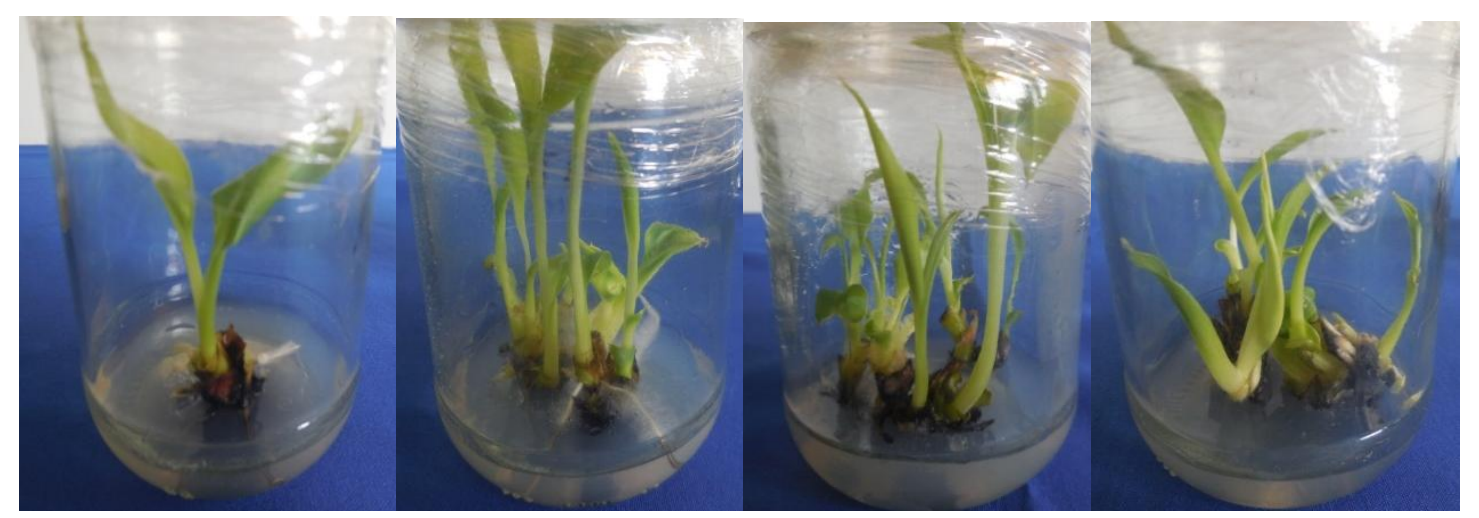

Figure 1. Shoot growth on media containing different kinds of cytokinins: A) Control; B) BAP ; C) BAP + NAA; D) TDZ.

\section{Fresh weight of plantlets}

The maximum fresh weight of explant (3374.80 mg/plantlet) was recorded with BAP $4 \mathrm{mg} / \mathrm{l}\left(\mathrm{T}_{3}\right)$ which was followed by BAP 6 $\mathrm{mg} / \mathrm{l}(3339.00 \mathrm{mg} / \mathrm{plantlet})\left(\mathrm{T}_{5}\right)$ and BAP 5 $\mathrm{mg} / \mathrm{l} \quad(3188.33 \mathrm{mg} / \mathrm{plantlet}) \quad\left(\mathrm{T}_{4}\right)$. While, minimum fresh weight of explants (1857.53 $\mathrm{mg} / \mathrm{plantlet}$ ) was recorded with untreated control $\left(\mathrm{T}_{1}\right)$ which was followed by TDZ $0.035 \mathrm{mg} / \mathrm{l}(2433.93 \mathrm{mg} / \mathrm{plantlet})\left(\mathrm{T}_{14}\right)$ and TDZ $0.030 \mathrm{mg} / \mathrm{l}(2496.93 \mathrm{mg} / \mathrm{plantlet})\left(\mathrm{T}_{13}\right)$.

\section{Per cent regeneration}

The maximum (100) per cent regeneration was observed with BAP $4 \mathrm{mg} / \mathrm{l}$ in both the subculture cycles (Table 1). Superiority of BAP may be due to the fact that it has a marked effect in stimulating the growth of auxiliary and adventitious buds and foliar development of shoot tip cultures. This result is in agreement with findings of Rahman et al., (2013) where best regeneration/ multiplication of shoots was observed in the MS medium containing $4 \mathrm{mg} / \mathrm{l} \mathrm{BAP}$ in banana cv. Agnishwar.

\section{Days taken for bud sprouting}

The time taken for bud sprouting was less with the medium containing BAP as compared to other treatments containing BAP in combination with NAA and TDZ (Table 2). Probably, this may be attributed to the greater effectiveness of BAP in promoting growth of axillary buds. A similar observation was also found from reports of Ahmed et al., (2014) in banana cv. Grand Naine.

\section{Number of shoots per explants}

The maximum number of shoots per explant was observed with BAP $4 \mathrm{mg} / \mathrm{l}$ (Table 3 ). The superiority of BAP is due to the fact that it is generally known to reduce the apical dominance and induce both axillary and adventitious shoot formation from multiple clump explants in banana. Similar findings were also reported by Muhammad et al., (2007) in banana cv. Basrai, Rahman et al., (2013) in banana cv. Agnishwar and Shankar et al., (2014) in banana cv. Grand Naine, who found incorporation of $4 \mathrm{mg} / \mathrm{l} \mathrm{BAP}$ in the media produced maximum number of shoots per explant. BAP has been reported to be in general the most active cytokinin for meristem shoot tip and axillary bud culture of various species (Maharana, 2012; Kar, 2015). Israeli et al., (1995), Mendes et al., (1996) and Strosse, et al., (2004) opined that shoot proliferation rate and elongation is significantly dependent on cytokinin type, its concentration and the genotype of banana cultivar. 


\section{Number of leaves per shoot}

The maximum number of leaves per shoot was produced on the medium supplemented with BAP $4 \mathrm{mg} / \mathrm{l}$ (Table 4). The result of the present experiment agrees with the findings of Rahman et al., (2004) who found that the maximum number of leaves (3.12/plantlets) was produced at 30 DAI with $5.0 \mathrm{mg} / \mathrm{l} \mathrm{BAP}$ in banana cv. BARI-1. Similar findings were also recorded by Ferdous et al., (2015) in which BAP $5.0 \mathrm{mg} / \mathrm{l}$ showed maximum number of leaves in banana cvs. Amritsagar and Sabri.

\section{Length of shoot}

In the present study, the longest shoot was obtained with BAP $4 \mathrm{mg} / \mathrm{l}$ (Table 5). The result of the present experiment agrees with the findings of Rahman et al., (2004) in banana cv. BARI-1, Rahman et al., (2013) in banana cv. Agnishwar and Uzaribara et al., (2015) in banana cv. Red banana who found that BAP containing media induced longer shoot length than other cytokinin types. The different results obtained by different authors might be due to differences of genotypes and explants used.

Variation in the activity of different cytokinins can be explained by their different uptake rate (Blakesley, 1991), varied translocation rates to meristematic regions and metabolic processes, in which the cytokinin may be degraded or conjugated with sugars or amino acids to form biologically inert compounds as reported by Kaminek (1992).

\section{Chlorophyll content}

The maximum chlorophyll content was observed with BAP $4 \mathrm{mg} / \mathrm{l}$ (Table 6). This may be due to the fact that cytokinin influence both chloroplast differentiation and chlorophyll biosynthesis. This is in accordance to Fletcher and McCullagh (1971) who found that etiolated cucumber cotyledons pretreated with BAP and exposed to light for 3 hours had up to $450 \%$ more chlorophyll than the water controls.

\section{Fresh weight of plantlets}

The fresh weight of the plantlet was high in MS medium supplemented with BAP (Table 7). Probably, this may be attributed to more number of shoots, long shoot length and maximum number of leaves induced by BAP $4 \mathrm{mg} / \mathrm{l}$. These results are in agreement with findings of Bhosale et al., (2011) in banana cvs. Ardhapuri, Basrai and Shrimanti and Sujin et al., (2016) in banana cv. Chenthuluvan where fresh weight of plantlets was higher in the media supplemented with BAP.

\section{Acknowledgement}

The authors are thankful to the University of Horticultural Sciences, College of Horticulture, Bagalkot, Karnataka, for their assistance in providing all the necessary facilities and equipment for conducting the research.

\section{References}

Ahmed, S., Sharma, A., Singh, A. K., Wali, V. K. and Kumari, P., 2014, In vitro multiplication of banana (Musa spp.) cv. Grand Naine. Afri. J. Biotechnol., 13 (27): 2696-2703.

Banerjee, N., Vuylsteke, D. and Langhe, D. E., 1986, Meristem tip culture of Musa: Histological studies of shoot bud proliferation. Plant tissue culture and its agricultural application (Withers, L. A. and Anderson, P. S., Eds). Butterworths, London, 139-148.

Bhosale, U. P., Dubhashi, S. V., Mali, N. S. and Rathod, H. P., 2011, In vitro shoot multiplication in different species of 
banana. Asian J. Plant Sci. Res., 1 (3): 23-27.

Blakesly, D., 1991, Uptake and metabolism of 6benzyladenine in shoot cultures of Musa and IBA pulsing for in vitro multiplication of banana cultivars through shoot-tip culture. J. Biosci. Agric. Res., 3: 87-95.

Ferdous, M. H., Billah, A. A. M., Mehraj, H., Taufique, T. and Uddin, A. F. M. J., 2015, BAP

Fletcherr, A. and McCullagh, D., 1971, Benzyladenine as a regulator of chlorophyll synthesis in cucumber cotyledons. Can. J. Bot., 49: 2197-2201.

Hirimburegama, K. and Gamage, N., 1996, In vitro multiplication of local cultivars of banana (Musa spp.) through shoot-tip culture. J. Nat. Sci. Council. 24: 9-20.

Israeli, Y., Lahav, E. and Reuveni, O., 1995, In vitro culture of bananas. In S. Gowen (Ed.), Bananas and plantains (pp.147178). London, U.K.: Chapman and Hall.

Kaminek, M., 1992, Progress in cytokinin research. Trends Biotechnol., 10: 159162.

Kar, D. K., 2015, In vitro seed culture of interspecific hybrid in Cymbidium spp. L. M.Sc. Ag. Thesis, OUAT, Bhubaneswar.

Maharana, D., 2012, In vitro shoot regeneration in hybrid cultivars of Gerbera (Gerbera jamesonii Bollus). M.Sc. Ag. Thesis, OUAT, Bhubaneswar.

Mendes, B. M. J., Mendes, F. J., Neto, A. T., Demetrio, C. G. B. and Puske, O. R., 1996, Efficacy of banana plantlet production by micropropagation. Pesquisa Agropecuária Brasileira. 31 (12): 863-867.

Muhammad, A., Rashid, H. and Hussain, I., 2007, Proliferation-rate effects of BAP and Kinetin on banana (Musa spp. AAA
Group) 'Basrai'. HortSci., 42:1253-1255.

Onuoha, I. C., Eze, C. J. and Unamba, C. I.N., 2011, In vitro prevention of browning in plantain culture. J. Bio. Sci., 11: 13-17.

Rahman, M. Z., Nassiruddin, K. M., Amin, M. A. and Islam, M. N., 2004, In vitro response and shoot multiplication of banana with BAP and NAA. Asian J. Plant Sci., 3: 406-409

Rahman, S., Biswas, N., Hassan, M. M., Ahmed, M. G., Mamun, A. N. K., Islam, M. R., Moniruzzaman, M. and Haque, M. E., 2013, Micro propagation of banana (Musa spp.) cv. Agnishwar by in vitro shoot tip culture. Int. Res. J. Biotechnol., 4 (4): 8388.

Rathod V., 2013, Studies on in vitro mutagenesis in banana. MSc. Hort. Thesis, University of Horticultural Sciences, Bagalkot. pp. 20.

Rhododendron. Plant Cell Tiss. Org. Cult., 25: 69-74.

Salaria, A. S., 2004, In: Horticulture at a Glance. Foreword by: Singh, K., Jain Brothers, New Delhi. 1: 131.

Singh, H. P., 2009, Research and development in banana and plantain-National and international scenario. In: Banana new innovations. Edited by Singh, H. P. and Mustaffa, M. M., Westville Publishing House, New Delhi. pp. 1.

Strosse, H. I., Houwe, V. D. and Panis, B., 2004, Banana cell and tissue culture: Review. In Jain, S. M. and Swennen, R. (Eds.). Banana improvement: cellular, molecular biology and induced mutations. (pp. 112). Polymouth, U.K.: Science Publishers Inc.

Uzaribara, E., Nachegowda, V., Ansar, H., Sathyanarayana, B. N. and Taj, A., 2015, In vitro propagation of Red banana (Musa acuminata). Bioscan. 10 (1): 125-129.

\section{How to cite this article:}

Aman, T., G. Prabhuling, K. Hipparagi, D.P. Prakash and Babu, A.G. 2018. In vitro Multiplication of Banana CV. Rajapuri Bale (Musa spp., AAB Group) Int.J.Curr.Microbiol.App.Sci. 7(07): 3141-3151. doi: https://doi.org/10.20546/ijcmas.2018.707.367 